\title{
Robust graphene-based monoliths of homogeneous ultramicroporosity
}

by

Teresa J. Bandosz ${ }^{1,2^{*}}$, Shuwen Wang ${ }^{1}$, Daiki Minami ${ }^{1}$ and Katsumi Kaneko ${ }^{1}$

${ }^{1}$ Shinshu University

Center for Energy and Environmental Science, 4-17-1 Wakasato, Nagano, 380-8553 Japan

${ }^{2}$ Permamet address: Department of Chemistry, The City College of New York, New York, NY 10031

*Whom the correspondence should, be addressed to. Tel.: (212)650-6017; Fax: (212) 650-6107; Email: tbandosz@ccny.cuny.edu 


\section{ABSTRACT}

Graphite oxide $(\mathrm{GO})$ and graphene monoliths were prepared using the unidirectional freezing of GO water suspension. These materials were saturated with a poly(ammonium-4-styrene sulfonate) water soluble polymer and then carbonized at $1123 \mathrm{~K}$. This process increases significantly the materials strength and density. A uniform deposition of the polymer-derived carbon on the external layers of the graphene sheets of the monolith was found. The carbon from polymer not only provided more contact between the graphene sheets but also apparently increased the overall graphitization level (based on Raman spectra). The modification decreased the electrical resistance by one order of magnitude compared to that of the graphene monolith. $\mathrm{N}_{2}$ adsorption at $77 \mathrm{~K}$ showed that the thus-treated graphene monoliths have quite homogenous pores with the pore width of $0.7 \mathrm{~nm}$. These pores, combined with large transport pores, and conductive properties make the monoliths tested the promising materials for separation, energy storage, and/or gas sensing. The tunability of the properties and pore structure of the robust graphene untramicroporous monolith through the control of chemistry of the initial GO monolith was shown.

\section{Introduction}

Carbon monoliths of specific performance have been actively studied for various applications including separation [1] and gas storage [2]. It is because because the monolith form has intriguing advantages over powdered activated carbons such as high electrical and thermal conductivities, high density storage, no intergranular barriers, and easy handling. For these applications the sizes of pores and their volumes are of paramount importance. Carbon monoliths are usually obtained using either binders [1] or in binder free process [2-4]. The latter one 
involves the extrusion method used for organic polymers. Applying binders reduces the performance of an active carbon phase per unit volume and might result in some instability of a monolith for longer applications. Another method is based on template carbonization and an example is a recently reported monolith obtained using porous concrete [5]. Nevertheless, in this approach a post-carbonization additional activation need to be applied to develop the microporosity. Another method is based on soft templates and usually triblock copolymers as, for instance, Pluronic F127 are used as templates [6,7]. Recently developed approach is based on unidirectional freeze-drying method for aqueous colloidal system without any binders and it was applied to develop monoliths from polymers [8] or graphite oxides [8]. Freeze -thaw cycles have been also proposed as a method of graphite oxide exfoliation [9].

Although the development of porosity in monoliths is essentially important, the methods proposed lead to materials that are not superior to activated carbons in this aspect [3-8]. Of course the shape and mechanical stability of a monolith is essential for industrial applications where powdered activated carbon are rather not desired. This causes that the efforts on the development of monoliths of interesting properties continue.

An advance in the graphene science has directed the attention of the scientist interested in monolith developments to this family of materials. Monoliths based on graphene can find new cutting edge-applications owing to the high electrical and thermal conductivity of graphene layers. Examples are energy storage devices and thermal isolators. Recently an ice-templating derived graphene based monolith of superporosity of $2200 \mathrm{~m}^{2} / \mathrm{g}$ and high electrical conductivity has been described by Kaneko and coworkers [8]. Unfortunately, even though the dispersive forces between binder free "self assembled" graphene sheets are strong enough to provide a predetermined shape, the structure is rather weak. 
The objective of this paper is to develop a new type of graphene-based monolith with very homogenous porosity in the range of ultramicropores being promising for separation technology. In their structure the integrity of the graphene layers is preserved providing the conductive properties. They are obtained using a saturation of the graphite oxide monolith with watersoluble commodity polymer followed by its carbonization. Application of this water -soluble polymer is an environmentally friendly aspect of the method propose. This simple treatment has provided a novel graphene-based monolith of quite uniform nanoporosity in addition to the mechanical strength.

\section{Experimental}

\subsection{Monolith coating procedure}

GO monoliths were obtained from 1:1 mixture (by weight) of GO obtained from Bay carbon graphite (Michigan, USA) and Madagascar graphite (obtained using Hummers method [10]). The details on the preparation of GO are described elsewhere [8]. Dense suspensions/pastes of GO were frozen with liquid nitrogen for $2 \mathrm{~h}$ and freeze dried at $223 \mathrm{~K}$ and $10 \mathrm{~Pa}$ for three days. The brownish GO monolith (60 $\mathrm{mm}$ in length ad $10 \mathrm{~mm}$ in diameter) obtained in this process is referred to as MGO. A subsample of the graphite oxide monolith. MGO, was partially reduced into a graphene monolith by treatment in argon at $623 \mathrm{~K}$ with heating rate $1.4 \mathrm{deg} / \mathrm{min}$ and holding time $30 \mathrm{~min}$. This sample is referred to as MR. Both MGO and MR were soaked in 15 wt \% aqueous solution of poly(4-ammonium styrene sulfonate) (MW: 200,000; Aldrich; referred to as ASSA) polymer for four hours. MR saturated with the polymer was then dried in room temperature for three days (denoted as MRP) and MGO saturated with the polymer was freeze-dried for three days (denoted as MGOP). Freeze-drying was done at the same conditions as those applied originally to GO monolith. Then the MGOP sample was reduced at $623 \mathrm{~K}$ at the 
same conditions as the initial GO and it is referred to as MPR. All three samples, MRP, MPR along with MR were heated at $1123 \mathrm{~K}$ in argon with a heating rate $30 \mathrm{deg} / \mathrm{min}$ and soaking time 1 hour. The final samples are referred to HT-MRP, HT-MPR and HT- MR following the treatment procedures. To better visualize the experimental steps applied the treatment procedure and the samples names are presented in a schematic way in Figure 1.

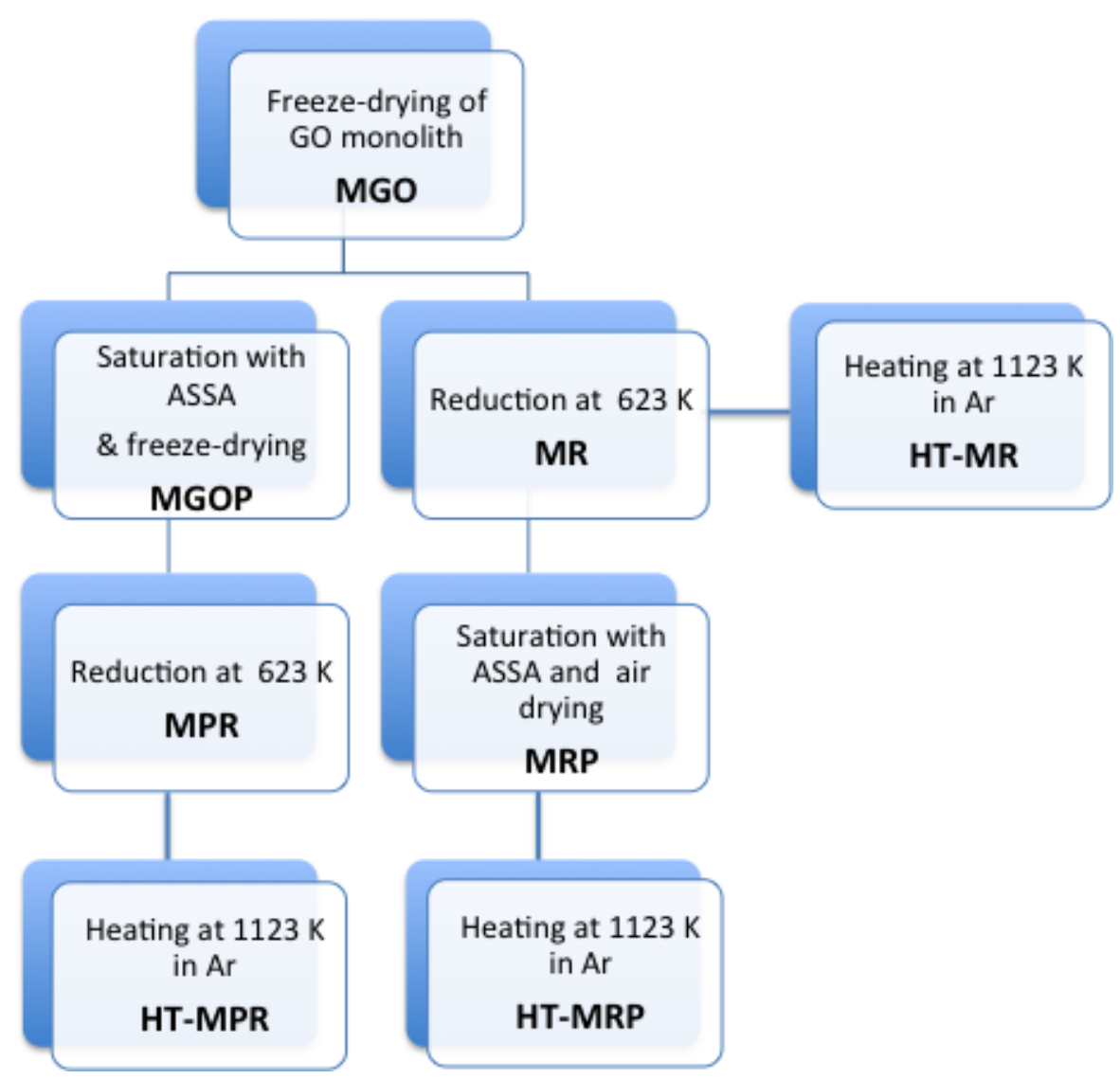

Figure 1. Schematic representation of the reinforced monolith preparation. 
For elucidate clearly the role of the carbon phase the ASSA polymer was carbonized in argon with two steps heating: 1) at $623 \mathrm{~K}$ (heating rate $1.4 \mathrm{deg} / \mathrm{min}$ ) with holding time $30 \mathrm{~min}$ and 2) at $1123 \mathrm{~K}$ in argon with the heating rate $30 \mathrm{deg} / \mathrm{min}$ and holding time $30 \mathrm{~min}$. The sample is referred to as PC.

\section{2. Characterization}

\subsubsection{Determination of apparent density}

The estimation of the monolith density was performed by measurement of their sizes (diameter and length) and weights.

\subsubsection{Electrical resistance}

Electrical resistance was measured on the external surface of monoliths using a four-point probe method (Leresta-GP MCP-T610; Mitsubishi). The resistance was measured along the monolith length (L) and on the cross section (D) surfaces.

\subsubsection{Optical microscope}

The optical microscope images were obtained on Olympus DP73 in a bright mode with the low magnification of $5 \mathrm{k}$ owing to the high degree of surface roughness.

\subsubsection{FTIR}

FTIR measurement was carried out using a Nicolet 6700 spectrometer using the attenuated total reflectance (ATR) method with a diamond crystal for the powdered samples without $\mathrm{KBr}$ addition. The spectrum was generated and collected 32 times and corrected for the background noise.

\subsubsection{TA/MS analysis}

Thermogravimetric (TG) curves were obtained using a Rigaku TG-DTA-PIMS 410/S, equipped with a mass spectrometer. The GOM and composite samples were heated up to $1123 \mathrm{~K}$ (10 deg 
$\left.\min ^{-1}\right)$ under a helium flow $\left(300 \mathrm{~mL} \mathrm{~min}^{-1}\right)$. The composition of released gases was evaluated by MS and m/z (mass-to-charge ratio) evolution profiles as a function of temperature were obtained.

\subsubsection{Raman spectroscopy}

The Raman spectra were obtained on a Renishaw InVia Raman spectrometer fitted with microscope and a $5 \times$ objective using a $532 \mathrm{~nm}$ laser with $1 \%$ power and exposure time $10 \mathrm{~s}$.

\subsubsection{SEM and TEM}

SEM images were collected on JEOL JSM 700F/IV field scanning emission electron microscope and JEOL-JSM-6335FS with $20 \mathrm{kV}$ and $10 \mathrm{kV}$ voltage applied, respectively. High resolution TEM images were collected on a high resolution transmission electron microscope (JEM-40000 FX, JEO).

\subsubsection{Evaluation of porosity}

Nitrogen adsorption isotherms were measured at $77 \mathrm{~K}$ on ASAP2020 (Micromeritics). Samples were out-gassed at $523 \mathrm{~K}$ for two hours. The surface areas and the volumes pores were determined. The former was obtained using the BET and Subtracting Pore Effect (SPE) methods [11]. The pore volumes along with the pore size distributions were obtained using Density Functional Theory approach with 2-D NLDFT model assuming heterogeneous surface of pore walls (www.NLDFT.com) [12].

\subsubsection{Testing of mechanical strength}

The evaluation of mechanical strength was done by weights of $20 \mathrm{~g}, 50 \mathrm{~g}$ and $100 \mathrm{~g}$ on the initial and modified monoliths.

\section{Results and Discussion}

3.1. Morphological and mechanical properties of the monoliths. 
The pictures of the obtained monoliths are presented in Figure 1S of Supplementary Information. As seen, as a result of the treatment applied the color and the geometrical dimensions of the monoliths changed. A slight shrinkage was observed. More importantly, the samples treated with the polymer became much harder than HT-MR. The initial MGO is a very soft and easy to disintegrate material. It was hypothesized that dispersive forces are responsible for the arrangement of GO particles during the rapid freezing and slow drying process [9]. Its texture resembles that of the paper filters of cigarettes with extended graphene oxide flakes arranged with the axial length of the monolith (freezing direction). Reduction at $623 \mathrm{~K}$ makes the structure slightly more stable, but it still remains very "fabric-soft". During this process a slow decomposition of epoxy groups present on the basal planes [13] results in an increase of dispersive interactions between the distorted graphene sheets. These interactions are even more enforced when the GO is reduced at $1123 \mathrm{~K}$. Nevertheless, the soft texture of the monolith remains after that high temperature treatment. Saturation of GO with the polymer, followed by drying, results in a visible shrinkage of the monoliths (about 30 volume \%).

The samples were exposed to various external weights to quantify the differences in the mechanical strength of MGO and HT-MR, and the polymer enforced monoliths (Figure 2). The MGO and HT-MR could be tested only when the force was applied horizontally to the monolith length since the disintegration was observed with any attempts to place monoliths in a free-stand position. For GOM and HT-MR visible dents/recesses are seen directly under a weight when 20 $\mathrm{g}$ and $50 \mathrm{~g}$ weights were applied, respectively. On the other hand, HT-MRP shows a remarkable strength and no changes were noticed even when $100 \mathrm{~g}$ was applied in both vertical and horizontal directions of the monolith. The tolerable maximum pressure indicates that the sample 
can maintain the external pressure of at least $30 \mathrm{kPa}$ without any visible sign of a structural disintegration.

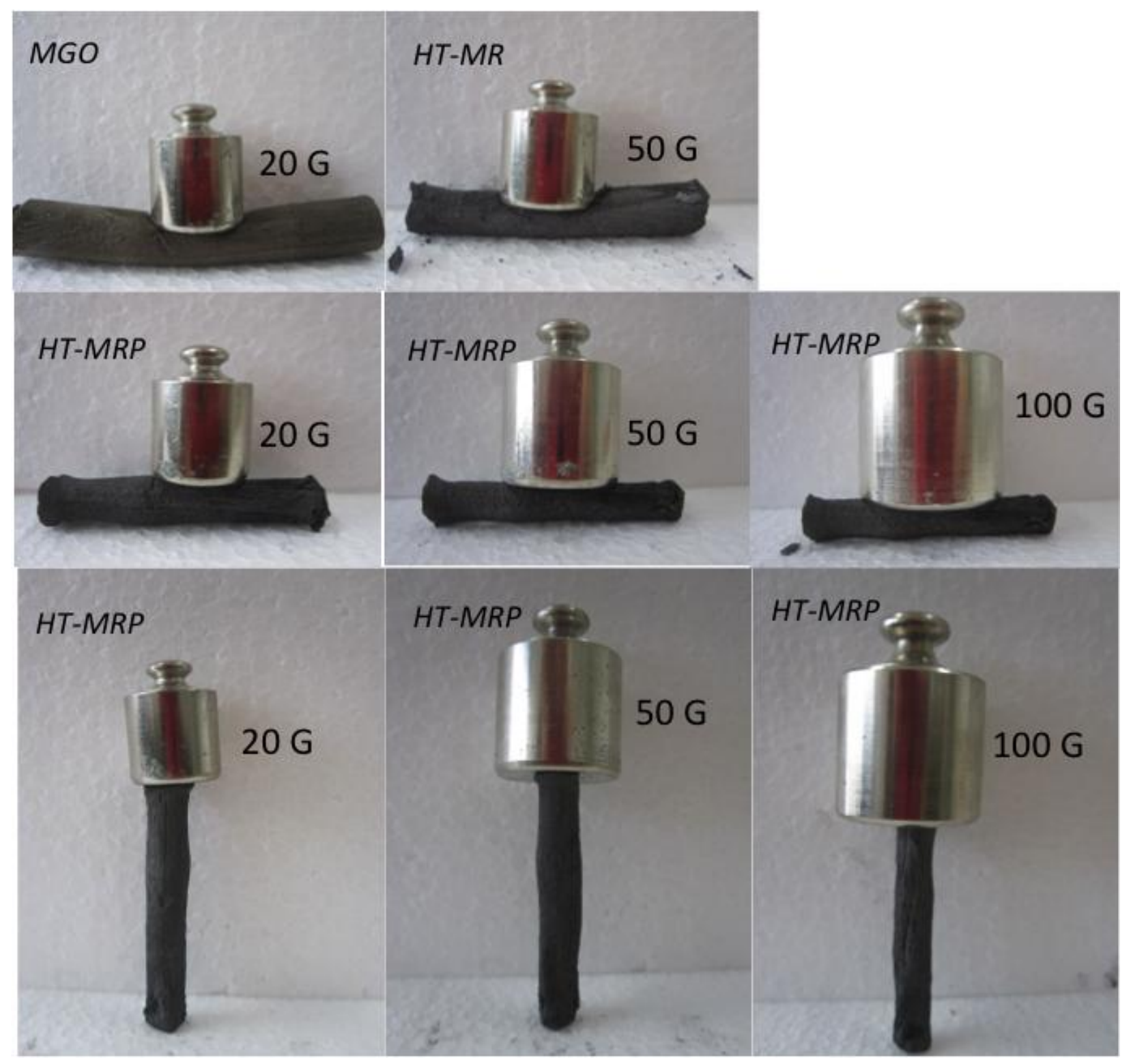

Figure 2. Comparison of the strength of the initial and modified monoliths. The diameter of HTMRP monolith is $7 \mathrm{~mm}$. 
The MGO sample gains about 3.5 times of its original weight as a result of the polymer saturation and freeze-drying. The last process visibly removed some polymer solution from the monolith volume. The reduced monolith MR adsorbed even more polymer (12 times of its original mass). It is likely owing to air drying and to that fact that reduction removed epoxy groups resulting in a more hydrophobic nature of graphene sheet and thus in a more favorable environment for adsorption of aromatic rings of the polymer via $\pi-\pi$ interactions. The detailed analysis of the changes in the sample weight at different steps of the preparation is presented in Supplementary Information.

\subsection{Nanoscopic morphology}

Optical images of the external surfaces of the HT-MR, HT-MPR and HT-MRP are presented in Figure $2 \mathrm{~S}$ of Supplementary Information. The composites visibly exhibit a denser arrangement of carbons grains than the HT-MR caused by the deposition of the polymer-derived carbon phase. A further insight into the texture is provided by SEM images collected in Figures 3 and 4. More images of various magnifications are included in Figure 3S of Supplementary informationThe surfaces of MGO and HT-MR consist of loosely connected, twisted and wrinkled flakes of GO of about $20 \mu \mathrm{m}$ in diameter and $10 \mathrm{~nm}$ thick (Figure 3). Those loose connections, even though they provide enough strength to maintain the integrity of the composites, must affect negatively the electronic conductivity of the monolith. Addition of the polymer totally changes the texture. In Figure 4 the surface of MPR is presented where the polymer is visible in a thermally changed but still not in a fully carbonized form. Here the flake of GO embedded in the polymer is clearly seen. The arrangement suggests that the polymer exhibits a very high adhesion to GO and the thickness of its layer is almost equal on the both sides of the graphene flake (about $500 \mathrm{~nm})$. The charred polymer texture follows a wrinkled surface of the GO flakes. 


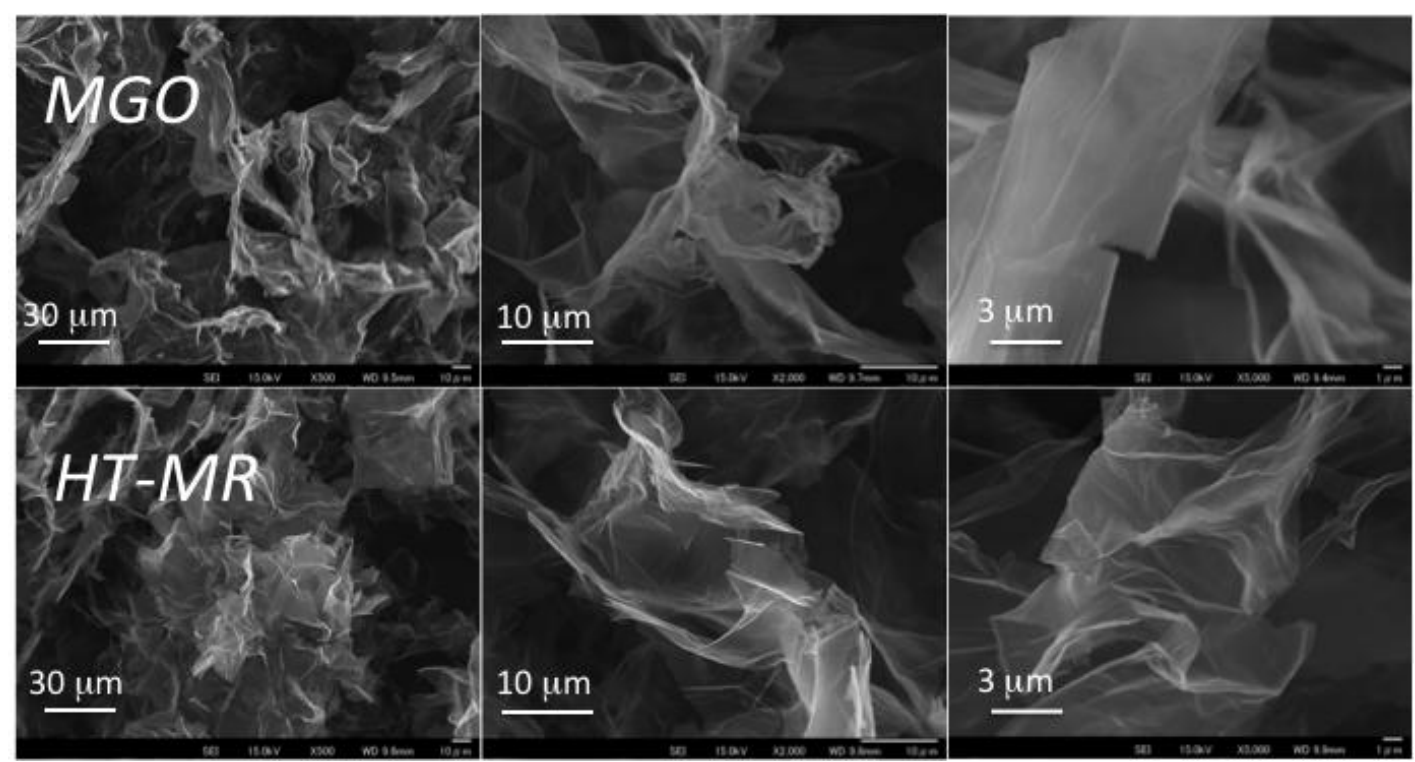

Figure 3. SEM images for MGO and HT-MR.

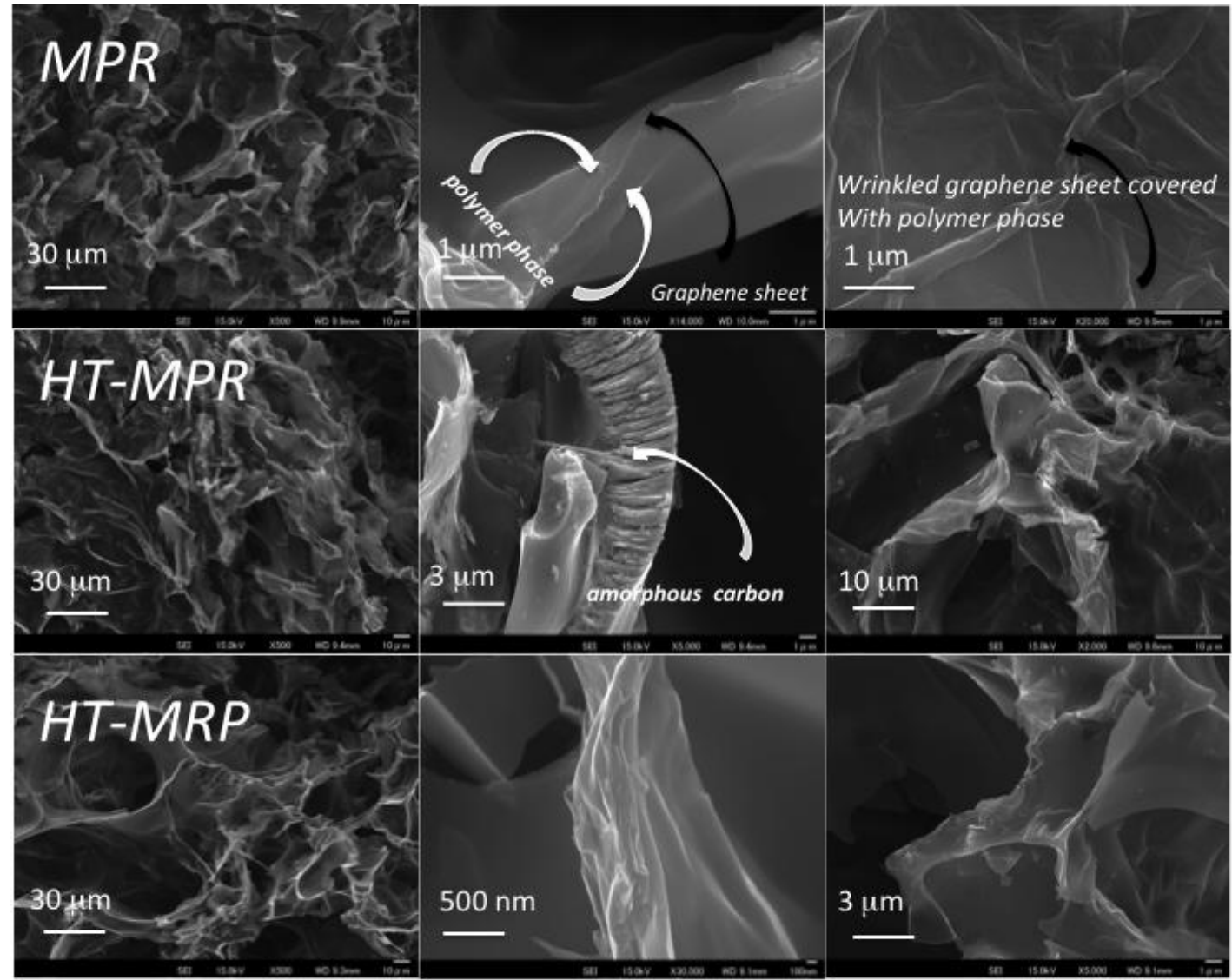


Figure 4. SEM images for the polymer treated monoliths,

There are visible differences in the texture of HT-MRP and HT-MPR with the latter sample exhibiting a denser texture with smaller particles. The thickness of polymer-derived carbon "walls" in the case of HT-MRP is about $200 \mathrm{~nm}$. In the case of HT-MPR the polymer-originated phase seems to be more bulky even reaching $2 \mu \mathrm{m}$ in its thickness with visible cracks in the amorphous carbon phase. This might be the result of the reaction of the polymer with the GO surface and formation of a specific resin that is more thermally stable than is the polymer itself [14. 15].

HRTEM images for the reinforced monoliths are presented in Figure 5. The images of HT-MRP show the presence of graphene layers, graphene covered with the carbon and the amorphous carbons phase. On the other hand, for HT-MPR the unmodified graphene layers cannot be detected, which supports the formation of a resin on their surface as a result of the reactions of GO functional groups with the sulfonic groups of the polymer $[14,15]$. This resin is converted into the amorphous carbon phase during the carbonization process and it covers the GO sheets. 


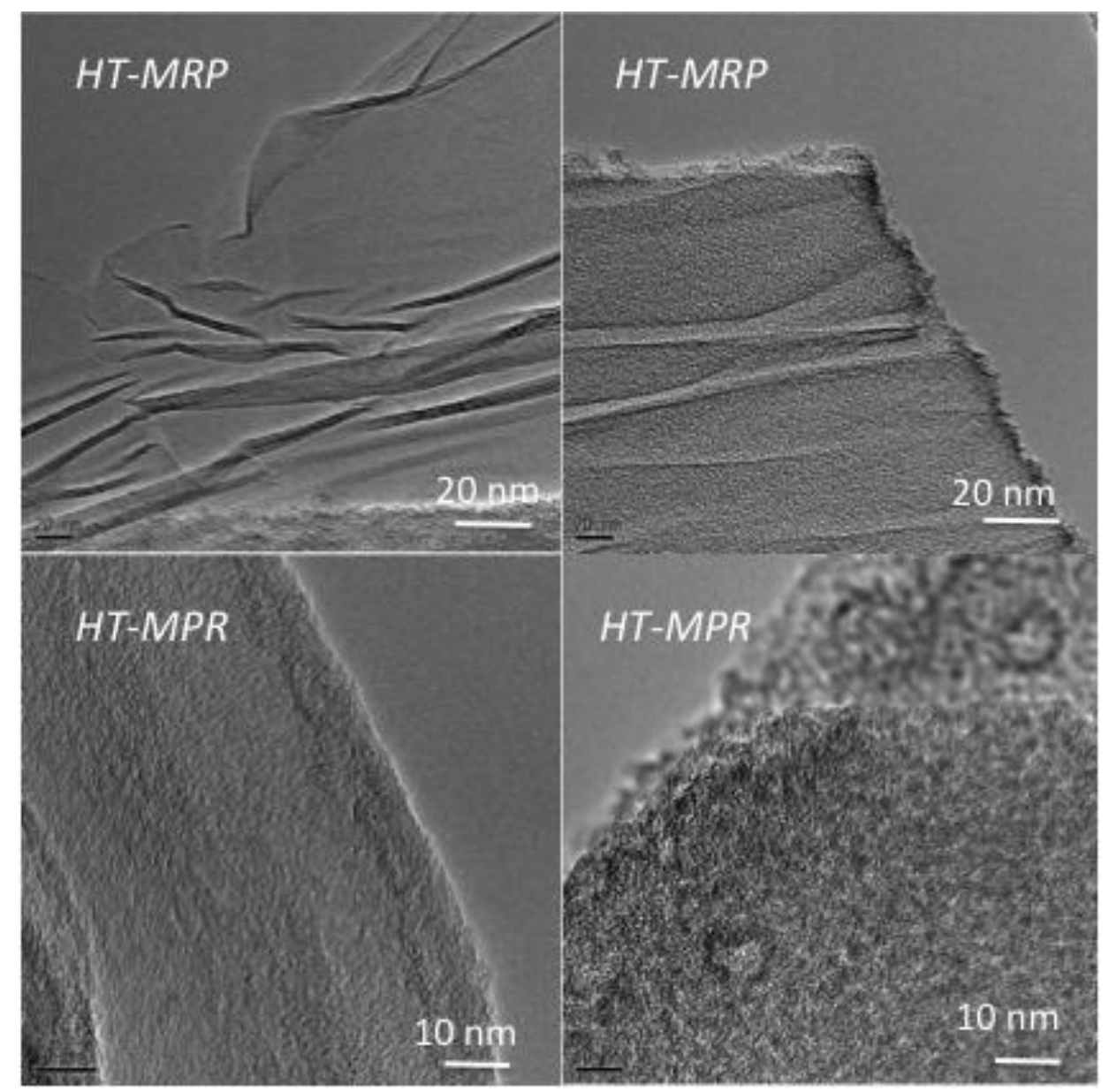

Figure 5. HRTEM images of the monolith texture.

\subsection{Chemical and physical properties of monoliths}

Although the experimental procedure is very simple, the polymer embedded in GO gives a sophisticated alteration of the monolith structure. During the saturation with the polymer especially in the case of the unreduced GO monolith, MGO, the reactions of sulfonic groups of the polymer with epoxy groups of the GO in the acidic environment can take place resulting in the formation of sulfonic acid ester bound to the graphene sheet surface $[14,15]$. A detailed analysis of the changes in the weight of the monolith during its preparation is provided in Supplementary Information. The results suggest that a significant amount of the partially charred 
polymer bound to the surface and thus thermally stable is still present within the structure of the MPR sample. This phase apparently follows different carbonization pattern than that exhibited by ASSA (Figure 4S of SI).

Heating the polymer-treated samples at $1123 \mathrm{~K}$ resulted in the monoliths of considerable physical strength though brittle when cut. The results indicate that even though the high temperature treatment was applied to MR leading to the reduction of the oxygen groups of low thermal stability in the form of $\mathrm{CO} / \mathrm{CO}_{2}$, the monolith still contains a significant amount of oxygen groups, likely $\mathrm{OH}$, which are expected to decompose at high temperatures [16]. Interestingly, the extent of the weight loss for the polymer-treated monolith is not in an agreement with the difference in the content of the polymer, which was adsorbed in greater quantity by MR than by the MGO sample (Supplementary Information). Apparently the initial reaction of the polymer with $\mathrm{GO}$ and the heat treatment at $623 \mathrm{~K}$ give rise to the composite material formed at the interface of both components as a results of chemical reactions which decomposes at higher temperatures than that obtained by a direct carbonization of the polymer adsorbed on the reduced MR monolith. In fact, more of the charred polymer phase was present on MPR treated at $623 \mathrm{~K}$ than that on MRP treated at the same conditions. Moreover, in the presence of the polymer, not only oxygen is removed form the surface but also nitrogen and sulfur caning functional groups 
Table 1. Electrical resistance, apparent density $\rho$ and the ratios of the $\mathrm{D} / \mathrm{G}$ band intensity from the Raman spectra. The resistance was measured along the monolith length (L) and on the cross section (D) surfaces.

\begin{tabular}{|l|l|l|l|l|}
\hline Sample & R-L $[\Omega]$ & R-D $[\Omega]$ & $\left.\rho \square \square \mathrm{g} / \mathrm{cm}^{3}\right]$ & $\mathrm{I}_{\mathrm{D}} / \mathrm{I}_{\mathrm{G}}$ \\
\hline MGO & $1.7 \times 10^{7}$ & $4.0 \times 10^{6}$ & 0.042 & 0.84 \\
\hline MR & 50 & 90 & 0.016 & 1.06 \\
\hline HT-MR & 14 & 13 & 0.015 & 1.09 \\
\hline HT-MRP & 5.5 & 4.4 & 0.091 & 1.00 \\
\hline HT-MPR & 1.2 & 1.6 & 0.169 & 1.07 \\
\hline PC & $5.5^{*}$ & NA & NA & --- \\
\hline
\end{tabular}

*resistance measured on the solid piece of the carbonized polymer of about $4 \mathrm{~cm}^{2}$

The evaluation of materials' density was carried out based on the estimated geometrical sizes of the samples and their weight. The results are collected in Table 1. Significant differences are seen. MGO is a very light material with the density of $0.042 \mathrm{~g} / \mathrm{cm}^{3}$. Obviously there is a large volume of void spaces between the aligned GO paper/fabric-like sheets. Reduction at $623 \mathrm{~K}$ decreases the density almost three times as a result of the removal of a significant amount of oxygen and carbon in the form of $\mathrm{CO} / \mathrm{CO}_{2}$. Then further heating decreases the density only slightly ( $10 \%)$. Addition of the polymer-based phase to both MGO and MR visibly increases the density of the monoliths, which apparently increases their strength and mechanical resistance. The densest material is obtained when the initial monolith, MGO, is treated with the polymer. It happens in spite of the fact that the amount of the polymer gained by the sample was much greater for the MR monolith. Apparently hypothesized above reaction of the monolith surface with the polymer reflected in the changes in the thermal decomposition pattern that led to the denser product. Another reason for these differences might be in the kinetics of the polymer decomposition and the effects of the released gases on the porosity development in the polymer based carbon. Thus in the case of HT-MRP, the fast released gases from the decomposition of 
the polymer could expand the GO layers resulting in large voids seen in the outer surface of the monolith (Figure 1S). On the other hand, during the slow heating to $623 \mathrm{~K}$ of the polymer present in MGOP the release of gases was much less rapid. This process was stopped and the partially charred polymer was stabilized at $623 \mathrm{~K}$ before undergoing a fast pyrolysis to $1123 \mathrm{~K}$ leading to an increased degree of benzene rings condensation. Thermal analysis and the changes in the polymer chemistry during heating clearly show a significant mass loss at about $623 \mathrm{~K}$ (Figure 4S). This effect can be related to the decomposition of sulfonic acid and removal of ammonia (see the polymer chemical formula in Figure 4S of Supplementary Information). Then, at about $773 \mathrm{~K}$ aromatization of the aromatic rings should take place with the removal of hydrocarbons [19]. The fast removal/decomposition of sulfonic groups and ammonia might affect in a negative way the development of porosity, if the polymer is present on the surface of the GO layers in an unchanged form. On the other hand, the decomposition of epoxy groups during heating at $623 \mathrm{~K}$ in the presence of the decomposing polymer should contribute to the porosity development in the char/carbon phase. This is not expected be the case for the HT-MRP sample.

Table 1 shows that the reduction of the GO monolith at $623 \mathrm{~K}$ significantly decreased the electrical resistivity (five orders of magnitude when measured in D-direction and six orders of magnitude when measured in L-direction) as a result of the removal of oxygen groups [15]. Then the resistance further decreased of one order of magnitude after carbonization at $1123 \mathrm{~K}$. The carbon coating caused an order of magnitude improvement in the monolith conductive properties. It has been reported that the carbon from the same polymer had a high electrical conductivity due to the unique structure consisting of $10 \mathrm{~nm}$ graphitic domains [17]. The obtained highly electrical conductive properties are very important for an application of these monoliths for energy storage 
[18] or as catalysts for oxygen reduction [19] or water splitting processes where the fast electron transfer is a desired feature [20]. Interestingly, the resistivity values measured for a specific sample are comparable through both dimensions, especially for the monoliths heated at $1123 \mathrm{~K}$ indicating the presence of conductive paths in various directions of the monoliths' volumes.
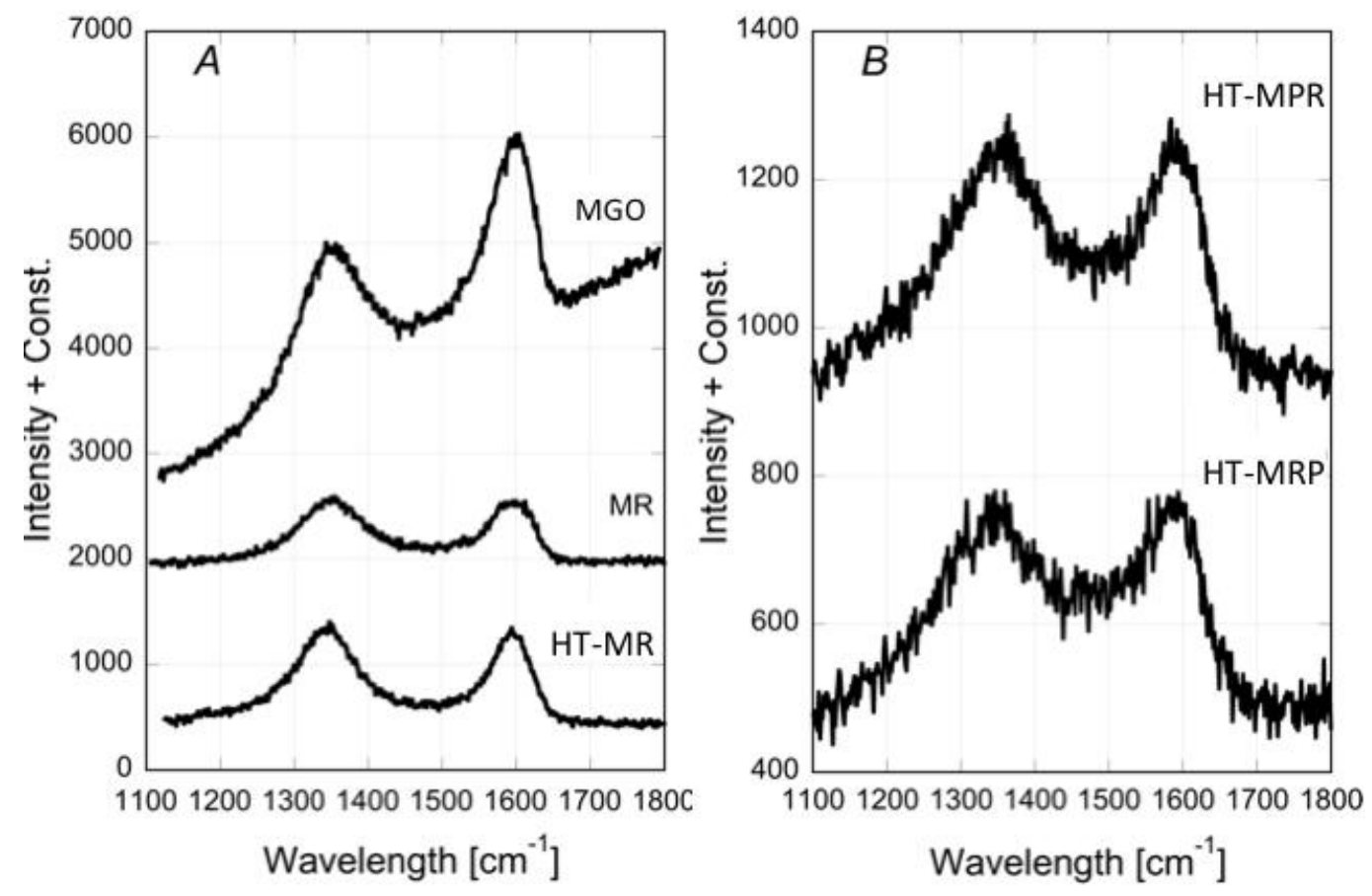

Figure 6. Raman spectra for the series of the graphene-based (A) and carbon-reinforced monoliths (B).

Raman spectra are collected in Figure 6. A distinct pair of absorption bands around $1350 \mathrm{~cm}^{-1}$ (D band) and $1580 \mathrm{~cm}^{-1}$ (G band) is visible for all samples. The $\mathrm{G}$ band and $\mathrm{D}$ band are assigned to the hexagonal carbon plane and crystal defects or imperfections in these planes, respectively [21]. Moreover, the ratio of the relative intensity of these two bands $\left(I_{D} / I_{G}\right)$ (Table 1) is considered as a measure of the number of defects in the carbonaceous materials [21]. The lower ratio indicates the higher graphitization level. Interestingly, the thermal treatment of the monolith increased the 
defects in the monolith as seen in an about $20 \%$ increase in $\mathrm{I}_{\mathrm{D}} / \mathrm{I}_{\mathrm{G}}$ for the modified samples in comparison withe that for MGO. This was likely caused by the removal of oxygen groups in the form of $\mathrm{CO} / \mathrm{CO}_{2}$. Since the change in the electrical resistance is greater than the changes in $\mathrm{I}_{\mathrm{D}} / \mathrm{I}_{\mathrm{G}}$, apparently the former parameter is much more sensitive to the oxygen removal than is the defectiveness level of the carbon frame observed by changes in $\mathrm{I}_{\mathrm{D}} / \mathrm{I}_{\mathrm{G}}$ from a Raman spectrum. The carbon coating decreases the $\mathrm{I}_{\mathrm{D}} / \mathrm{I}_{\mathrm{G}}$ ratio for HT-MRP compared to that for HT-MR. This is an indication that the carbon phase increases the overall level of graphitization in this material This trend is also consistent with an increase in the monolith electrical conductivity. Interestingly, the change in upon an introduction of the carbon phase for HT-MPR is much smaller than that found for HT-MRP

\subsection{Mechanism of the carbon reinforcement of the monoliths}

The FTIR spectra for the graphene monoliths are presented in Figure 7. The detailed assignment of absorption bands is presented in Supplementary Information. While for the MGO the typical bands representing oxygen groups at $1040 \mathrm{~cm}^{-1}, 1225 \mathrm{~cm}^{-1}, 1360 \mathrm{~cm}^{-1}, 1620 \mathrm{~cm}^{-1}$, and $1720 \mathrm{~cm}^{-}$

1 are visible [22], after the treatment at $1123 \mathrm{~K}$ only residual oxygen bonded to carbon is seen on the spectra for all samples at $1600 \mathrm{~cm}^{-1}$ representing quinones [16, 22$]$. These results indicate that even though the polymer has been introduced to the monolith and carbonized, it does not change the infrared absorption spectrum of the graphene based matrix. Moreover, sulfur, oxygen and nitrogen in the polymer originally present in the polymer do not give the apparent absorption bands inherent to their surface functional groups. Thus the thermal treatment likely removed the majority of heteroatoms from the carbon phase. The reason for that significant reduction can be also in a reducing effect of the graphene phase itself. On the other hand, the spectrum for the 
polymer-derived carbon PC shows multiple peaks coming from the abundance of oxygen groups, which suggests the low level of aromatization/carbonization [23].
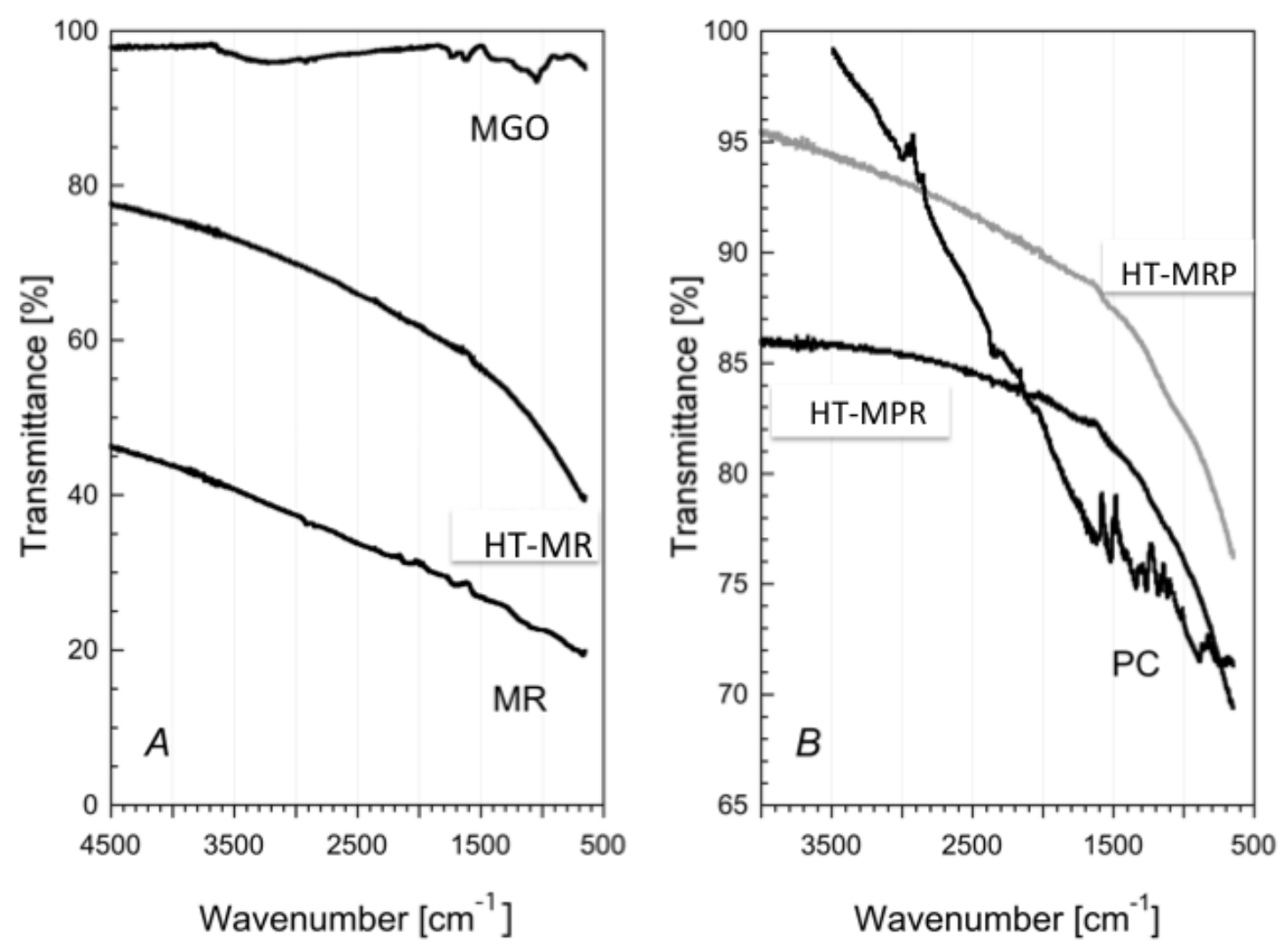

Figure 7. FTIR spectra for the series of the graphene based (A) and carbon reinforced monoliths and the polymer derived carbon (B).

The thermal analysis results for the graphene based monoliths and polymer-enforced counterparts are collected in Figures $6 \mathrm{~S}$ of Supplementary Information. They confirm the reduced nature of the graphene-based monolith as a result of high temperature treatment and the decomposition of surface oxygen groups [16]. The m/z thermal profiles for MR and HT-MR show the similarities in the removal pattern of surface decomposition products (Figure $6 \mathrm{~S}$ and $7 \mathrm{~S}$ 
of Supplementary Information), although the intensities of the current for the latter sample are at least three orders of magnitude smaller than those for the former one.

Thermal analysis data for the polymer treated monoliths show slight differences in materials thermal stability/surface chemistry (Figure 8 B). Both samples are very stable and lose only up to $3.5 \%$ mass. The differences observed weight loss even after treatment at $1123 \mathrm{~K}$ suggest that HT-MPR is slightly less reactive with the atmospheric oxygen than the sample obtained from the reduced monolith saturated with the polymer, HT-MRP. It is plausible to assume that the surface chemistry of the monolith has an important effect on the properties of the carbon phase and the surface bounded phase, which might form on the surface of the MGO sample. It apparently leads to the more stable carbon phase than that in the case of the reinforced monolith which was obtained from the reduced GO saturated with the polymer. 

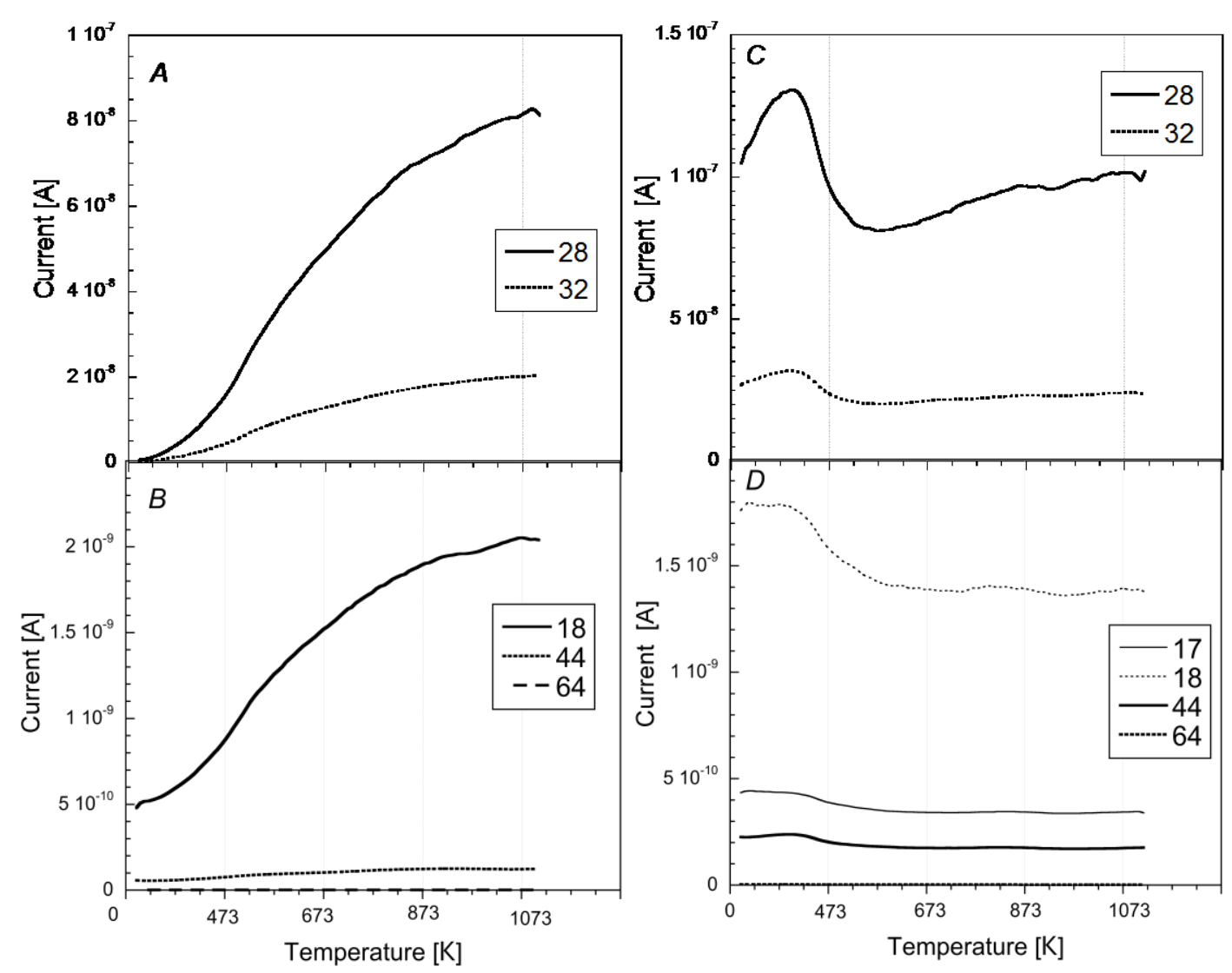

Figure $8 . \mathrm{m} / \mathrm{z}$ ( numbers presented in the legends) thermal profiles for HT-MPR (A and B) and HT-MRP (C and D). The profiles for m/z 64 are of low intensity and they overlap with the Xaxis.

$\mathrm{m} / \mathrm{z}$ (mass-to-charge ratio) thermal profiles for HT-MRP are different than those for HT-MPR (Figure 8,, Figure 8S), which supports the hypothesis presented above on the effect of GO surface chemistry on the final properties of the monolith. For the former sample the profiles show the removal of oxygen $(\mathrm{m} / \mathrm{z} 32)$ and decomposition of carboxylic groups (as water (m/z 17 and 18$), \mathrm{CO}(\mathrm{m} / \mathrm{z} 28)$, and $\left.\mathrm{CO}_{2}(\mathrm{~m} / \mathrm{z} 44)\right)$ at low temperatures suggesting higher reactivity of this monolith's carbon phase.. On the other hand, on the surface of HT-MPR rather basic OH groups, 
quinones or carbonyls decompose at high temperatures [16]. Those totally different thermal $\mathrm{m} / \mathrm{z}$ patterns suggest that the different carbon phases are present in these two monoliths. They also differ from those for the carbon from the polymer (Figure 9S of Supplementary Information). The results support the hypothesis presented above that epoxy groups of GOM could react with sulfonic moieties of the polymer forming a specific resin/coating on the surface $[14,15]$. Threedimensional comparison of $\mathrm{m} / \mathrm{z}$ profiles for the samples studied are collected in Figure $8 \mathrm{~S}$.

\subsection{Porous properties}

The measurements of nitrogen adsorption isotherms revealed that MGO, MR and HT-MR are the nonporous monoliths with the surface area not exceeding $3 \mathrm{~m}^{2} / \mathrm{g}$. On the other hand, the carbon reinforced monoliths exhibit porous structure (Table 2, Figure 9 A and B). Interestingly, the carbon derived from the polymer itself does not show any porosity which once again distinguishes it from the carbon described in Ref. [17] were a small surface area, $38 \mathrm{~m}^{2} / \mathrm{g}$, was reported. Apparently, carbonization conditions are an important factor determining the properties of the final products. Besides the surface area evaluated with the BET method, which is not accurate for very microporous materials [24], the surface area was also evaluated using SPE method [11]. Moreover, the pore volume and surface area were obtained using 2D-NLDFT assuming the heterogeneous surface of pore walls [12]. The BET surface area is smaller than the surface area from SPE and DFT methods, indicating that the pores are ultramicropores [11]. It is well known that BET analysis overestimates the surface area, because the adsorption data in the relative pressure of 0.05 to 0.35 includes the double layer adsorption induced by an overlapped interaction potential. Therefore, the Subtracting Pore Effect method (SPE) was used. It can extract the adsorption from monolayer process on the pore walls to evaluate an accurate surface are for micropores in the range $0.7-2 \mathrm{~nm}$. For this a high-resolution $\alpha_{\mathrm{s}}$ plot, which corresponds to 
the adsorption data in the range of relative pressure $10^{-6}$ to 1 , is applied. Regardless the method, the HT-MPR is more porous than HT-MRP. Its surface area and volume of micropores are 21 and $17 \%$ greater than those for the latter sample.
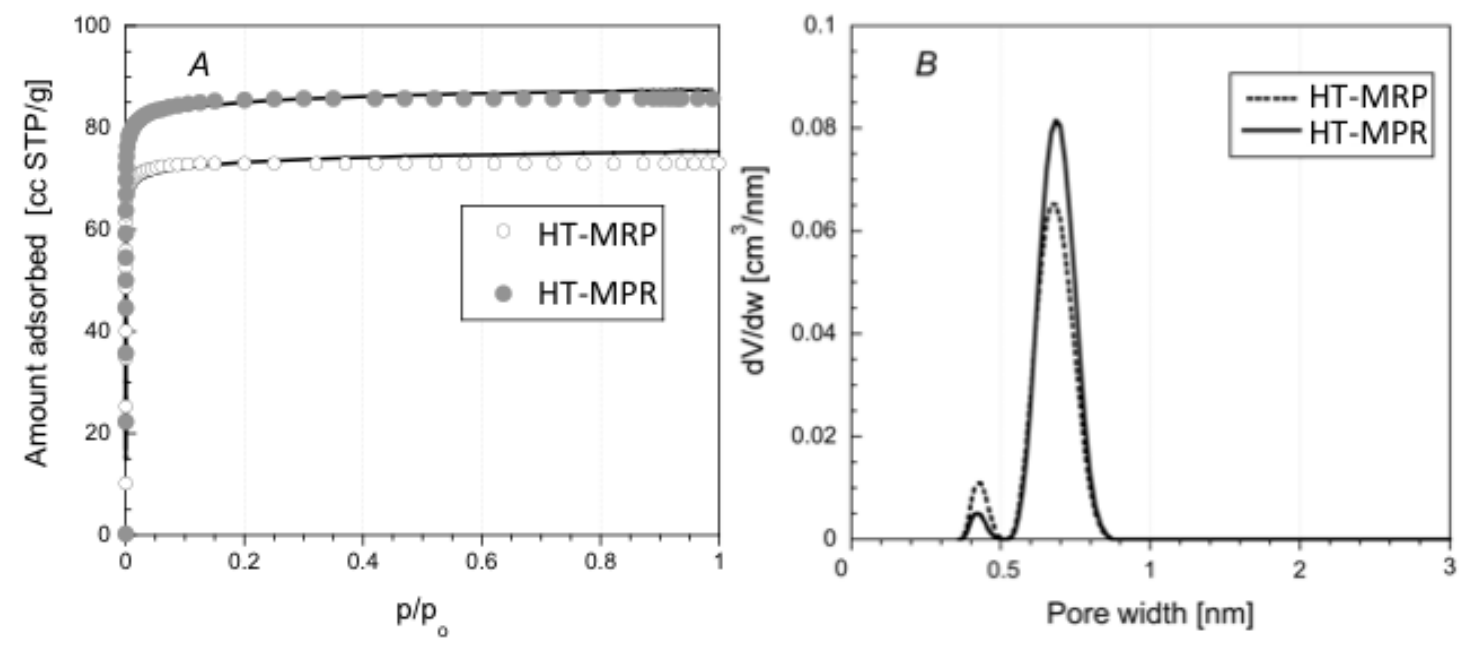

Figure 9. Nitrogen adsorption isotherms with the fit to NLDF (A) and pore size distributions (B) for the carbon reinforced graphene monoliths. 
Table 2. The parameters of porous structure determined from the nitrogen adsorption isotherms.

\begin{tabular}{|l|l|l|l|l|l|}
\hline Sample & $\begin{array}{l}\mathrm{S}_{\text {BET }} \\
{\left[\mathrm{m}^{2} / \mathrm{g}\right]}\end{array}$ & $\begin{array}{l}\mathrm{S}_{\mathrm{SPE}} \\
{\left[\mathrm{m}^{2} / \mathrm{g}\right]}\end{array}$ & $\begin{array}{l}\mathrm{S}_{\mathrm{DFT}} \\
{\left[\mathrm{m}^{2} / \mathrm{g}\right]}\end{array}$ & $\begin{array}{l}\mathrm{V}_{<\mathrm{nm}}=\mathrm{V}_{\mathrm{t}} \\
{\left[\mathrm{cm}^{3} / \mathrm{g}\right]}\end{array}$ & $\begin{array}{l}\mathrm{V}_{<0.5 \mathrm{~mm}} \\
{\left[\mathrm{~cm}^{3} / \mathrm{g}\right]}\end{array}$ \\
\hline HT-MRP & 229 & 345 & 328 & 0.107 & 0.007 \\
\hline HT-MPR & 277 & 415 & 363 & 0.122 & 0.003 \\
\hline
\end{tabular}

Slow kinetics of the adsorption isotherm measurements indicated the presence of ultramicropores. The pore size distribution from the 2D-NLDFT indicates evidently the presence of ultramicropores whose width is less than $0.7 \mathrm{~nm}$ in both samples, as presented in Figure $9 \mathrm{~B}$. Both monoliths can be considered as molecular sieves but HT-MPR is more homogeneous in the pore sizes. That $0.7 \mathrm{~nm}$ size of pores is identical for both samples implying the presence of an optimum interaction between the graphene sheets and newly introduced carbon. The higher volume of pores in HT-MPR that that in HT-MRP is linked to the higher content of the porous carbon phase in the former monolith. Interestingly, a slightly higher porosity of HT-MPR is accompanied by its almost twice higher density that those of HT-MRP. This might result in higher mechanical robustness of the former sample, which is important in real life applications of this material. Moreover, these characterization results indicate the importance of the polymerfunctional groups of GO interactions for the final properties of the monoliths.

\section{Conclusions}

This paper shows the procedure leading to the production of relatively rigid graphene based monoliths having highly uniform ultramicroporosity. The process of monolith reinforcement is environmentally friendly and uses a water soluble polymer with no washing of the final material required. The ultramicropores are uniformly developed in the monolith form, leading to efficient transport channels for molecules and ions. At the same time, the robust monolith has no 
interparticle gaps, offering low electrical resistance. The developed monoliths have high application potentials for separation/molecular sieving and selective storage of gases and ions. The deposition of the porous phase on the conductive graphene based frames of an interconnected network with large void space will provide not only a fast electron transfer but also an easy access of the electrolytes to those pores where the most efficient electrical double layer charge storage is possible [25]. Another application, which requires the $0.7 \mathrm{~nm}$ pores of high volume, is a selective separation of $\mathrm{CO}_{2}$ with the relevance to $\mathrm{CO}_{2}$ sequestration [26, 27]. As this robust graphene monolith can be applied to a gas filter, we can sense small gas molecules on filtering using the electrical conductivity change for monitoring local environments.

\section{Acknowledgement}

The authors are grateful to Professor Morinobu Endo for supporting experimental studies. TJB expresses here gratitude to Drs. Toshihiko Fujimori, and Ryusuke Futumura for their patience and help with instrumental approaches and to Dr. Rodolfo Cruz-Silva for his experimental help in collecting SEM images.

This work was supported by Grant-in-Aid for Scientific Research (A) (24241-38) and ConcertJapan project: Efficient Energy Storage and Distributions, JST. The sabbatical staying of TJB on Shinsu University was funded by the new functioned carbon membrane project. 


\section{REFERENCES}

1. Muhammad, Choong TSY, Chuah TG, Yunus R, Yap YHT. Adsorption of $\beta$-carotene onto mesoporous carbon coated monolith in isopropyl alcohol and n-hexane solution: equilibrium and thermodynamic study, Chem Eng J 2010; 164: 178-182

2. Silvestre-Albero A, Ramos-Fernández J M, Martínez-Escandell M, SepúlvedaEscribano A, Silvestre-Albero J, Rodríguez-Reinoso F, High saturation capacity of activated carbons prepared from mesophase pitch in the removal of volatile organic compounds. Carbon 2010;48: 548-556.

3. Kubo T, Sakamoto H, Fujimori T, Itoh T, Ohba T, Kanoh H, Martinez-Escandell M, Ramos-Fernandez J M, Casco M, Rodriguez-Reinoso F, Urita K, Moriguchi I, Endo M, Kaneko K, Diffusion-barrier-free porous carbon monoliths as a new form of activated carbon. ChemSusChem 2012; 5:2271-2277.

4. Elaigwu SE, Greenway GM. Biomass derived mesoporous carbon monoliths via an evaporation-induced self-assembly. Mater Lett 2014;115:117-120.

5. Zhao X, Zhang Q, Chen CH-M, Zhang B, Reiche S, Wang A, Zhang T, Schlogl R. Su DS. Aromatic sulfide, sulfoxide, and sulfone mediated mesoporous carbon monolith for use in supercapacitor. Nano Energy 2012; 1: 624-630.

6. Wen P, Gao J, Zhang Y, Liu Y. Fabrication of chitosan scaffolds with tunable porous orientation structure for tissue engineering. J Biomater Sci, Polym Ed 2011;20:19-40.

7. Zhang N, Qiu H, Si Y, Wang W, Gao J. Fabrication of highly porous biodegradable monoliths strengthened by graphene oxide and their adsorption of metal ions. Carbon $2011 ; 49: 827-37$.

8. Wang S, Tristan F, Minami D, Fujimori T, Cruz-Silva R, Terrones M, Takeuchi K, Teshima K, Rodriguez-Reinoso F, Endo M, Kaneko K. Activation routes for high 
surface area graphene monoliths from graphene oxide colloids. Carbon 2014; 76: 220 -231 .

9. Ogino I, Yokoyama Y, Iwamura S. Mukai SR Exfoliation of Graphite Oxide in Water without Sonication: Bridging Length Scales from Nanosheets to Macroscopic Materials. Chem Mater 2014;26:3334-3339.

10. Hummers, W. S.; Offeman, R. E., Preparation of Graphitic Oxide. J Am Chem Soc 1958, 80: 1339-1339.

11. Kaneko K, Jshi C, Ruike M, Kubawara H. Origin of superhigh surface area and microscystalline graphitic c structures of activated carbons. Carbon 1992; 30; 10751088.

12. Jagiello J, Olivier JP. Carbon slit pore model incorporating surface energetical heterogeneity and geometrical corrugation. Adsorption 2013; 19:777-783

13. .Huh SH. Thermal reduction of graphene oxide. In: Mikhailov S, editor. Physics and applications of graphene - experiments, InTech; 2011 p. 73-90.

14. Blank WJ. Isocyanate modified blocked sulfonic acid ester as a crosslinking catalyst. US patent EP0377931 B1 (1993).

15. McDonald MD. Metal coating compositions. US patent 2,885,312 (1959).

16. Figueiredo JL, Pereira MFR, Freitas MMA, Órfão JJM. Modification of the surface chemistry of activated carbons. Carbon 1999;37:1379-89.

17. Bandosz TJ, Castellon-Rodriguez E, Montenegro JM, Seredych M. Photoluminescence of nanoporous carbons: Opening a new application route for old materials Carbon 2014; 77: 651-659

18. Endo M, Maeda T, Takeda T, Kim YJ, Koshiba K, Hara H, Dresselhaus MS. 
Capacitance and pore-size distribution in aqueous and nonaqueous electrolytes using various activated carbon electrodes. J Electrochem Soc 2001;148:A910-4.

19. Liang J, Jiao Y, Jaroniec M, Qiao SZ. Sulfur and nitrogen dual-doped mesoporous graphene electrocatalyst for oxygen reduction with synergistically enhanced performance. Angew Chem Int Ed 2012;51:1-6; Angew Chem 2012;124:11664-8.

20. Xie G, Zhang K, Guo B, Liu Q, Fang L, Gong JR. Graphene-based materials for hydrogen generation from light-driven water splitting. Adv Mater 2013;25:3820-39.

21. Kudin, K. N.; Ozbas, B.; Schniepp, H. C.; Prud'homme, R. K.; Aksay, I. A.; Car, R., Raman Spectra of Graphite Oxide and Functionalized Graphene Sheets. Nano Letters 2007, 8 (1), 36-41.

22. Zawadzki J. IR spectroscopy in carbon surface chemistry. In: Thrower PA, editor. Chemistry and physics of carbon, vol 21, New York; Dekker; 1989 p. 180-200.

23. Seredych M, Koscinski M, Sliwinska-Bartkowiak M, Bandosz TJ. Charge storage accessibility factor as a parameter determining the capacitive performance of nanoporous carbon-based supercapacitors. ACS Sustainable Chem Eng 2013;1:10241032.

24. Marsh H, Rodriguez-Reinoso F. Activated Carbon. Elsevier, Amsterdam. 2005.

25. Chmiola J, Yushin G, Gogotsi Y, Portet C, Simon P, Taberna P.L. Anomalous increase in carbon capacitance at pore sizes less than 1 nanometer. Science 2006;313:1760-1763.

26. Wickramarante NP, Jaroniec M. Importance of small micropores in $\mathrm{CO}_{2}$ capture by phenolic resin-based activated carbon spheres. J Mater Chem A 2013:1 : 112-116.

27. Seredych M, Jagiello J, Bandosz TJ. Complexity of on nanoporous $\mathrm{CO}_{2}$ adsorption on 
sulfur-doped carbons - Is surface chemistry an important factor? Carbon 2014; 74:207-2017. 


\section{Captions to the Tables}

Table 1.

Electrical resistance, estimated density and the ratios of the intensity of band representing defects to the band representing $\mathrm{sp}^{2}$ configurations for the samples studied. The resistance was measured along the monolith length (L) and on the cross section (D) surfaces.

\section{Table 2.}

The parameters of porous structure determined from the nitrogen adsorption isotherms. 


\section{Captions to the Figures}

Figure 1. Schematic representation of the reinforced monolith preparation.

Figure 2. Comparison of the strength of the initial and modified monoliths. The diameter of HTMRP monolith is $7 \mathrm{~mm}$.

Figure 3. SEM images for MGO and HT-MR.

Figure 4. SEM images for the polymer treated monoliths

Figure 5. Figure 5. HRTEM images of the monolith texture.

Figure 6. Raman spectra for the series of the graphene-based (A) and carbon-reinforced monoliths (B).

Figure 7. FTIR spectra

Figure 8. $\mathrm{m} / \mathrm{z}$ ( numbers presented in the legends) thermal profiles for HT-MPR (A and B) and $\operatorname{HT}-M R P(C$ and D).

Figure 9. Nitrogen adsorption isotherms with the fit to NLDF (A) and pore size distributions (B) for the carbon reinforced graphene monoliths. 